Jan RADIMSKÝ

Université de Bohême du Sud

\title{
SUPPORTS CENTRAUX ET SUPPORTS PÉRIPHÉRIQUES : DES « MAILLONS FAIBLES » DU LEXIQUE-GRAMMAIRE ?
}

\section{Introduction}

La présente contribution s'inscrit dans le cadre d'un projet de recherche, dont le but est de confronter le fonctionnement des prédicats nominaux à verbe support en tchèque, et dans deux langues romanes: en italien et en français. Pour le tchèque, la seule étude complexe sur ce sujet a été faite dans les années soixantedix du siècle dernier par Eva Macháčková. Sa thèse de doctorat, intitulée Syntagmes analytiques du type verbe + nom abstrait (expression analytique des

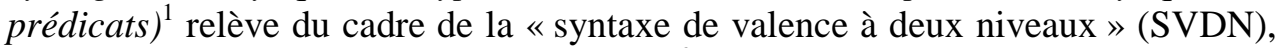
élaborée par František Daneš et son équipe. ${ }^{2}$ Dans les langues romanes, c'est dans le cadre du Lexique-Grammaire que la majeure partie des études sur ce sujet a été faite. Comme le Lexique-Grammaire, à l'heure actuelle, offre une base méthodologique plus élaborée que la SVDN, nous avons repris ses outils afin de les tester sur le matériel de la langue tchèque.

Dans notre recherche, nous avons modifié deux paramètres techniques par rapport à la méthodologie lexico-grammaticale. En premier lieu, le «test de l'acceptabilité », considéré comme le moyen principal de vérification par M. GROSS (1975 : 19-22), a été remplacé par un recours systématique aux grands corpus, le Corpus national tchèque en l'occurrence. En second lieu, nous partons du nom prédicatif pour rechercher les différents verbes supports qui peuvent l'actualiser, tandis que la méthode lexico-grammaticale procède dans le sens inverse.

Bien que les deux paramètres soient de nature technique, en ce sens qu'il n'imposent aucune modification dans la conception théorique du prédicat nominal dans le Lexique-Grammaire, leur application effective a permis de repérer du matériel qui, d'une certaine manière, nous amène à repenser le cadre théorique de départ. Dans la présente contribution, nous nous proposons d'analyser à l'aide du concept centre-périphérie (DANEŠ F., 1966) plusieurs paramètres de variabilité des verbes supports qui peuvent accompagner un nom prédicatif.

\section{La conception théorique}

L'un des avantages principaux du Lexique-Grammaire - par rapport à la SVDN par exemple - est qu'il offre un procédé formel pour définir le prédicat nominal à support. Il s'agit d'une transformation, au cours de laquelle le support, ainsi que d'autres outils grammaticaux, sont effacés, comme dans l'exemple suivant (cf. GROSS M., 1981) :

\footnotetext{
${ }^{1}$ MACHÁČKOVÁ E. (1979). Pour une revue plus détaillée des études sur ce sujet pour le tchèque voir RADIMSKÝ J. (2009b).

${ }^{2}$ Cf. DANEŠ F., HLAVSA Z. a kol. (1981).
} 
(1) Max a de l'affection pour Lucie.

(2) L'affection Max a pour Lucie.

(3) L'affection de Max pour Lucie.

Si l'on veut vérifier que le verbe avoir dans notre exemple ne remplit effectivement que des fonctions grammaticales, donc qu'il est effaçable sans perte de l'invariant sémantique, il faut vérifier qu'il est reconstructible en lisant la suite des transformations dans le sens inverse. Ainsi, lorsque nous contextualisons l'exemple (3) dans une phrase (4), l'invariant sémantique doit être conservé après l'adjonction du verbe support :

(4) L'affection de Max pour Lucie est surprenante.

(5) L'affection que Max a pour Lucie est surprenante.

En effet, les phrases (4) et (5) sont synonymes, l'invariant sémantique est conservé. Ce test, proposé initialement par Zellig S. HARRIS (1970), nous donne par le biais des notions de transformation et d'invariant sémantique un outil formel qui permet de distinguer l'emploi support de l'emploi prédicatif d'un verbe donné.

Observons à présent, comment ce type de prédication nominale est formellement décrit par Maurice Gross (1981) dans le cadre du LexiqueGrammaire. Une confrontation rapide avec le modèle proposé par la SVDN (MACHÁČKOVÁ E., 1979) nous permettra de mieux faire ressortir les propriétés de ce modèle.

Les deux tableaux qui suivent présentent un schéma de la représentation formelle de la phrase Max a de l'affection pour Lucie. La première description correspond au cadre de la SVDN, tandis que la seconde relève de la description suivant le Lexique-Grammaire.

Tableau 1-analyse par la SVDN

\begin{tabular}{|l|l|l|l|l|}
\hline \multirow{2}{*}{ Sujet } & \multicolumn{2}{|l|}{ Prédicateur } & \multicolumn{2}{|l|}{ Complément } \\
\cline { 2 - 5 } & $\begin{array}{l}\text { Centre de valence } \\
\text { syntaxique }\end{array}$ & $\begin{array}{l}\text { Prédicat } \\
\text { Centre de valence } \\
\text { sémantique }\end{array}$ & pour N1 \\
\hline NO & $a$ & de l' & affection & pon \\
\hline
\end{tabular}

Tableau 2 - analyse par le Lexique-Grammaire

\begin{tabular}{|l|l|l|l|l|}
\hline \multirow{2}{*}{$\begin{array}{l}\text { Argument } \\
\text { (sujet) }\end{array}$} & \multicolumn{2}{|l|}{ Actualisateur } & Prédicat & \multirow{2}{*}{ Argument N1 } \\
\cline { 2 - 4 } & Verbe support & & Prédicat nominal & \\
\hline NO & $a$ & de l' & affection & pour NI \\
\hline
\end{tabular}

Il est intéressant de constater à quel point les deux descriptions convergent. Dans les deux approches, le nom prédicatif est décrit comme «prédicat ». Quant aux différences entre les deux modèles, la SVDN met un peu plus l'accent sur la valence syntaxique du verbe support qui, combinée avec la valence sémantique du prédicat nominal, détermine la valence du prédicateur analytique dans son 
ensemble. Autrement dit, la SVDN a tendance à considérer le prédicateur analytique (avoir de l'affection) comme une unité complexe, tandis que le Lexique-Grammaire considère les deux unités séparément.

\section{Enjeux méthodologiques}

\section{III.1. Le point de départ de la recherche}

Le modèle du prédicat nominal proposé dans le cadre du Lexique-Grammaire (GROSS M., 1981) permet d'emprunter deux chemins différents lors de la recherche du matériel. L'une est de rechercher les prédicats nominaux correspondant à un support donné (6), l'autre est inverse (7).
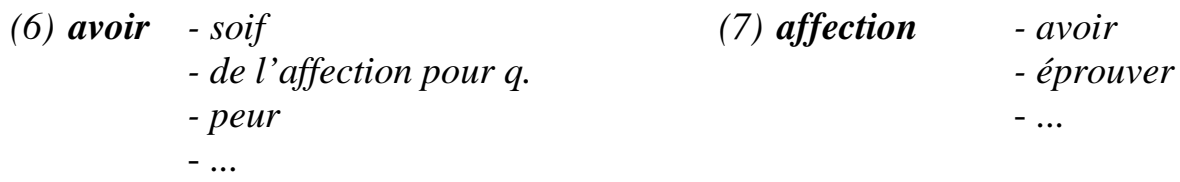

L'équipe de Maurice Gross a choisi la première solution, celle de partir d'un verbe support concret. Ce choix était probablement motivé par le fait qu'en français, le verbe support est l'élément le moins variable du système, en ce sens qu'une petite quantité de supports génériques (comme faire, donner, mettre, passer, prendre, avoir, être) sont susceptibles d'actualiser une quantité énorme de noms prédicatifs. Ainsi, la liste des verbes qui peuvent être employés comme « supports » en français s'allongeait progressivement - de 14 unités repérées par Anne Daladier en 1978, jusqu'à 98 unités citées par Gaston Gross en 1998 (IBRAHIM A. H., 2000).

Nous avons choisi la démarche inverse pour deux raisons principales.

1) Sur le plan théorique, le Lexique-Grammaire confère au nom prédicatif le statut de «prédicat», en privilégiant nettement la dépendance sémantique par rapport à la dépendance syntaxique. Il est donc légitime d'admettre que c'est le nom prédicatif - en tant qu'élément sémantiquement régissant - qui sélectionne son actualisateur, donc son verbe support. Par conséquent, il nous semble plus justifié de partir du nom (élément régissant) pour rechercher le verbe support (élément dépendant).

2) Il semble qu'en tchèque, la combinatoire des supports génériques soit beaucoup plus restreinte qu'en français. L'intuition laisse croire que la plupart des noms prédicatifs sont actualisés notamment par des supports spécifiques. C'est précisément ce qui a été constaté par Grażyna VETULANI (2000) dans sa recherche sur les prédicats nominaux en polonais; cet auteur arrive même à la conclusion qu'en polonais, pratiquement chaque verbe peut avoir un emploi «support » pour un nom prédicatif spécifique. Donc pour le tchèque comme pour le polonais, nous ne disposons pas d'une liste limitée de supports génériques de départ.

Notons sur ce point qu'une première application de la relation centrepériphérie se révèle : pour les sept verbes supports « génériques » français cités, la fonction support est beaucoup plus centrale que pour d'autres verbes, aussi bien au niveau du système (leur spectre combinatoire avec les noms prédicatifs est beaucoup plus large) qu'au niveau du discours (dans le discours, ils apparaissent 
plus fréquemment en fonction support par rapport à d'autres verbes). En réalité, de ce point de vue, le verbe support central par excellence serait en français procéder $\grave{a}+N$ (correspondant à provádět $+N$ en tchèque), car même s'il est moins fréquent que les précédents en chiffres absolus, il s'emploie presque exclusivement en fonction support (cf. Radimský J., 2010 : 137-139).

\section{III.2. L'introspection vs. le corpus}

Dans les Méthodes en syntaxe, Maurice Gross (1975:19-24) appuie sa méthode expérimentale sur la seule intuition du linguiste. Ce dernier devrait procéder au test d'acceptabilité des formes étudiées, afin de coder de manière binaire (+ / -) les constructions acceptables et inacceptables. Maurice Gross est conscient des difficultés et des incertitudes que la réduction binaire des faits linguistiques apporte, mais reste tout de même persuadé qu'une réduction binaire est possible et plausible. Dans le même ouvrage, Maurice Gross (1975 : 24) se prononce également contre l'usage des corpus dans le travail expérimental, en avançant, entre autres, l'argument selon lequel le corpus ne permet pas d'attester de nombreuses constructions acceptables.

Il serait superflu de reprendre ici les nombreux arguments qui, au fil de plusieurs décennies, ont progressivement fait placer le corpus au centre du travail expérimental en linguistique. ${ }^{3}$ Nous nous contenterons de présenter rapidement quelques conséquences importantes du recours systématique au corpus dans le domaine et dans le cadre théorique qui nous intéresse.

a) Le corpus permet de combler le fossé entre la «compétence » et la «performance » du chercheur, en lui permettant de repérer les constructions qu'il connaît, mais qu'il n'est pas capable de trouver lui même.

b) Le corpus atteste des constructions que le chercheur - n'étant jamais un locuteur «parfait »-considère comme inacceptables, notamment dans les langues de spécialité. ${ }^{4}$

c) Il est vrai que le corpus n'atteste pas certaines constructions acceptables, comme le souligne Maurice Gross. En effet, sur un corpus aussi grand que le SYN 2000 qui compte 100 millions de mots, certains supports accompagnant même les noms prédicatifs fréquents, sont assez rares, avec à peine des dizaines, voire des unités d'occurrences (cf. tableau 3). Par conséquent, nous pouvons imaginer que la taille et la représentativité des corpus des années 1970-1980 ne permettaient pas d'effectuer ce type de recherche.

d) Le codage binaire en construction acceptable / inacceptable peut être remplacé par un codage scalaire qui rend compte du nombre d'occurrences d'une forme.

\footnotetext{
${ }^{3}$ Cf. par exemple RADIMSKÝ J. (2005).

${ }^{4}$ Il ne s'agit pas nécessairement de langages techniques maîtrisés uniquement par quelques spécialistes. Les amateurs de foot par exemple - très nombreux dans la population maîtrisent couramment des constructions à verbe support très spécifiques (p. ex. nastřílet 5 gólů, vsítit gól - pour marquer un but) que la langue standard ignore.
} 
e) Le corpus nous fournit des exemples de contextualisation qui dépassent l'imagination active du linguiste. Ainsi, il est plus facile de juger si le test de l'effacement est positif.

Voici un exemple des supports repérés pour le nom prédicatif požadavek (exigence) dans le corpus SYN 2000. Les chiffres indiquent le nombre d'occurrences avec le nom prédicatif au singulier et au pluriel. Notons que le nom požadavek n'est pas un mot rare : dans le dictionnaire de fréquence EFES basé sur le corpus SYN 2000, il atteint la position 637, avec environ 17.000 occurrences.

Tableau 3. - actualisateurs du $N_{\text {préd. }}$ požadavek (exigence) dans le SYN 2000

\begin{tabular}{|c|c|c|c|c|}
\hline Vsup. + Npréd. & Vsup. & Fréq. Sg. & Fréq. Pl. & Total fréq. \\
\hline klást požadavek & klást (poser) & 16 & 163 & 179 \\
\hline mít požadavek & mít (avoir) & 12 & 80 & 92 \\
\hline vznést požadavek & vznést (faire envoler perf.) & 57 & 23 & 80 \\
\hline vyslovit požadavek & vyslovit (prononcer perf. $_{\text {) }}$ & 41 & 9 & 50 \\
\hline formulovat požadavek & formulovat (formuler) & 13 & 28 & 41 \\
\hline vznášet požadavek & vznášet (faire envoler ${ }_{\text {imperf. }}$ ) & 6 & 30 & 36 \\
\hline předložit požadavek & předložit (présenter ${ }_{\text {perf. }}$ ) & 11 & 24 & 35 \\
\hline přjijít s požadavkem & přjijít (venir avec perf. ) & 17 & 14 & 31 \\
\hline přednést požadavek & přednést (réciter perf.) & 7 & 8 & 15 \\
\hline vyslovovat požadavek & vyslovovat (prononcer ${ }_{\text {imperf. }}$ ) & 6 & 4 & 10 \\
\hline předkládat požadavky & předkládat (présenter ${ }_{\text {imperf.) }}$ ) & 0 & 9 & 9 \\
\hline přicházet s požadavkem & přicházet (venir avec imperf. $_{\text {) }}$ & 4 & 3 & 7 \\
\hline dát požadavek & dát (donner) & 3 & 2 & 5 \\
\hline vyrukovat s požadavkem & vyrukovat (« aller au régiment ») & 3 & 2 & 5 \\
\hline přednášet požadavek & přednášet (réciter ${ }_{\text {perf. }}$ ) & 1 & 2 & 3 \\
\hline
\end{tabular}

Au total, nous avons repéré 15 verbes supports différents et nous pourrions probablement en trouver encore quelques-uns, avec une fréquence basse. Examinons à présent les paramètres possibles de cette variabilité surprenante. En effet, les fréquences repérées laissent supposer que dans la construction avec le même nom prédicatif, certains supports seront plus centraux que d'autres.

\section{Paramètres de variabilité des supports}

\section{IV.1. Les paramètres décrits par le Lexique-Grammaire}

Déjà dans son article sur les Prédicats sémantiques de 1981, Maurice GROSS (1981 : 33) distingue les verbes supports simples et leurs variantes qu'il qualifie de « aspectuelles ». ${ }^{5}$ Dans un article plus récent (GROSS M., 1998), il passe en revue systématiquement l'apport sémantique du verbe support, en proposant ainsi une vue plus fine des paramètres de leur variabilité. En gros, nous pouvons regrouper ces paramètres en deux ensembles qui correspondraient à deux zones plus périphériques de la fonction «support » d'un verbe.

\footnotetext{
${ }^{5}$ P.ex. les variantes suivantes : L'argent a / prend / conserve / garde / perd de l'influence sur Max. (Gross M., 1981 : 33).
} 
Le premier paramètre affecte l'expression syntaxique des arguments sémantiques du prédicat. Il y a plusieurs restructurations possibles :

a) Support converse. Etudié notamment dans la thèse de Gaston Gross (1989), ce support permet d'inverser l'argument sujet et l'argument objet.

(8) Léa donne une gifle à Luc.

(9) Luc reçoit une gifle de Léa.

b) Support causatif. Il permet d'exprimer un argument externe en position sujet.

(10) Luc a du courage.

(11) Cette nouvelle a donné du courage à Luc.

c) Support «impersonnel ». Dans ce type de support, peu décrit par le LexiqueGrammaire (l'étiquette «impersonnel » est la nôtre ${ }^{6}$ ), le nom prédicatif revêt le rôle du sujet syntaxique, ce qui fait que tous les arguments peuvent généralement être omis. Cette construction peut être obtenue soit à l'aide d'un support d'occurrence (13), soit en passivant un support ordinaire (14).

(12) Le médecin a effectué l'examen du dossier.

(13) L'examen du dossier a déjà eu lieu.

(14) L'examen (du dossier) a été effectué.

Toutes ces restructurations peuvent être repérées également en tchèque. Voici, à titre d'exemple, une liste des constructions correspondantes pour le nom prédicatif požadavek (exigence). Ces constructions ne figuraient pas dans le tableau précédent.

Tableau 4. - actualisateurs converses, causatifs et impersonnels $d u N_{\text {préd. }}$ požadavek (exigence) dans le SYN 2000

\begin{tabular}{|c|c|c|c|c|c|}
\hline Type & Vsup. + Npréd. & Vsup. & $\begin{array}{l}\text { Fréq. } \\
\text { Sg. }\end{array}$ & $\begin{array}{l}\text { Fréq. } \\
\text { Pl. }\end{array}$ & $\begin{array}{l}\text { Total } \\
\text { fréq. }\end{array}$ \\
\hline \multirow[t]{2}{*}{ converse } & obdržet požadavek & obdržet (obtenir) & 2 & 1 & 3 \\
\hline & dostat požadavek & dostat (recevoir) & 6 & 6 & 12 \\
\hline causatif & vyvolat požadavky & vyvolat (provoquer) & 1 & 13 & 14 \\
\hline \multirow{5}{*}{ impersonnel } & požadavek vzejde & vzejít (émaner) & 5 & 1 & 6 \\
\hline & $\begin{array}{l}\text { požadavky se } \\
\text { objeví }\end{array}$ & objevit se (se montrer) & 19 & 9 & 28 \\
\hline & $\begin{array}{l}\text { požadavky se } \\
\text { množí }\end{array}$ & $\begin{array}{l}\text { množit se (se } \\
\text { multiplier) }\end{array}$ & 0 & 3 & 3 \\
\hline & požadavek existuje & existovat (exister) & 6 & 10 & 16 \\
\hline & požadavek přichází & přicházet (venir) & 1 & 2 & 3 \\
\hline
\end{tabular}

Le second paramètre que nous pouvons relever chez Maurice GROSS (1998) concerne les significations relevant du domaine du mode d'action (Aktionsart). En gros, ces significations s'articulent soit autour des phases du procès (15), soit autour de son intensité (16) :

${ }^{6}$ Cf. RADIMSKÝ J. (2009a). 
(15a) Luc (commence + continue + finit + poursuit) la lecture du texte

(15b) Luc a (recommencé + répété + réitéré) la lecture du texte.

(16) Ce rapport (déborde + grouille + fourmille + regorge) de bonnes idées.

Les deux ensembles que nous venons de décrire correspondent à deux zones périphériques de la fonction «support» d'un verbe. En effet, les verbes support converses et causatifs ne sont pas effaçables selon la même transformation que le support central ; la règle de la coréférence des sujets (GROSS M., 1981) ou « inaliénabilité » du sujet (CATTEL R., 1984), caractéristique des supports ordinaires, n'est pas respectée. Quant au second groupe, les supports qui expriment un mode d'action spécifique ne sont pas reconstructibles au cours du test de l'effacement ; l'invariant sémantique n'est pas complètement conservé lors de la transformation.

Seuls les supports du type «impersonnel» sont relativement proches du centre, en ce qu'ils admettent l'effacement :

(17a) Tyto požadavky mne znepokojily.

Ces exigences ont suscité mon inquiétude.

(17b) Tyto požadavky, které (se objevily + existovaly + přicházely + vzešly od NO) mne znepokojily.

Ces exigences qui (se sont montrées + existaient + venaient + émanaient de NO) ont suscité mon inquiétude.

\section{IV.2. Variantes stylistiques et « modalités spécialisées »}

Après avoir écarté ces zones périphériques il faut se poser la question de savoir si toutes les constructions restantes, c'est-à-dire «centrales », passent avec succès le test de l'effacement du support. En fait, cela revient à dire que dans le contexte d'une phrase, les différentes constructions support devraient être parfaitement interchangeables sans que l'invariant sémantique soit modifié, comme dans les exemples (19) et (20) :

(18) L'affection de Max pour Lucie est surprenante.

(19) L'affection que Max $\boldsymbol{a} \quad$ pour Lucie est surprenante.

(20) L'affection que Max éprouve pourLucie est surprenante.

Comme la recherche se fait sur corpus, nous pouvons vérifier la synonymie des différentes constructions à partir des contextes réels. En effet, nous pouvons vérifier dans le tableau 5, que parmi les 15 supports repérés pour le nom prédicatif požadavek (exigence), 13 sont interchangeables. Le seul facteur grammatical qui interdit de remplacer les supports utilisés dans les exemples ci-dessus par n'importe quel autre support de la colonne de gauche est l'aspect perfectif ou imperfectif, dicté par chaque contexte. Evidemment, l'expression syntaxique des différents arguments n'aura pas la même forme, mais les changements de cas et des prépositions nécessaires restent sans impact sur la conservation de l'invariant sémantique. 
Tableau 5. - exemples contextualisés selon SYN 2000

\begin{tabular}{|c|c|}
\hline Vsup. & Exemple \\
\hline dát (donner) & $\begin{array}{l}\text { K soudu jsme ale dali požadavek, aby k nám neposílali recidivisty, kteří nejsou } \\
\text { zvyklí pracovat, " říká tajemník [...]. }\end{array}$ \\
\hline $\begin{array}{l}\text { formulovat } \\
\text { (formuler) }\end{array}$ & $\begin{array}{l}\text { Generál Mladič při své osobní schůzce s Janvierem formuloval jasný } \\
\text { požadavek a Janvier se mu bez odporu podrobil : žádné nálety, pak budou } \\
\text { rukojmí propuštěni. }\end{array}$ \\
\hline $\begin{array}{l}\text { predkládat } \\
\text { (présenter }{ }_{\text {imperf.) }}\end{array}$ & Bude jedna strana pouze předkládat své požadavky a druhá je jen odmítat ? \\
\hline $\begin{array}{l}\text { předložit } \\
\text { (présenter } \\
\text { perf.) }\end{array}$ & $\begin{array}{l}\text { Ačkoliv jednotlivé výbory sněmovny předložily požadavky na téměř } \\
\text { miliardové navýšení, drtivá většina návrhủ byla vyvážena změnami ve vládní } \\
\text { rozpočtové rezervě. }\end{array}$ \\
\hline $\begin{array}{l}\text { přednášet (réciter } \\
\text { perf.) }\end{array}$ & $\begin{array}{l}\text { Živě si představil, jak Bob vytáčí číslo a bez slůvka vysvětlení přednáší svi̊j } \\
\text { požadavek. }\end{array}$ \\
\hline $\begin{array}{l}\text { přednést (réciter } \\
\text { perf.) }\end{array}$ & $\begin{array}{l}\text { [...] mluvčí OF přednesl požadavek, aby se o kooptaci nehlasovalo jednotlivě, } \\
\text { ale najednou. }\end{array}$ \\
\hline $\begin{array}{l}\text { přicházet (venir } \\
\text { avec }_{\text {imperf.) }}\end{array}$ & $\begin{array}{l}\text { Mezinárodní měnový fond přichází s požadavkem hospodářské reformy, jeho } \\
\text { podmínky jsou moc tvrdé, lidé se bouří. }\end{array}$ \\
\hline $\begin{array}{l}\text { přijít } \\
\text { (venir avec } \text { perf.) }\end{array}$ & $\begin{array}{l}\text { Po válce přišlo Japonsko s požadavkem navrácení alespoň těch ostrovů, jež pod } \\
\text { ruskou jurisdikcí nikdy dříve nebyly. }\end{array}$ \\
\hline $\begin{array}{l}\text { vyrukovat } \\
(\text { («aller au } \\
\text { régiment ») }\end{array}$ & $\begin{array}{l}\text { V New Yorku už bylo vše natočené a právě jsme pracovali v Londýně, když } \\
\text { Dino vyrukoval s požadavkem, abych některé scény z filmu škrtl. }\end{array}$ \\
\hline $\begin{array}{l}\text { vyslovit } \\
\text { ( } \text { prononcer }_{\text {perf. }} \text { ) }\end{array}$ & $\begin{array}{l}\text { Rakouská banka vyslovila požadavek, aby česká vláda tuto záležitost nějakým } \\
\text { způsobem řešila [...]. }\end{array}$ \\
\hline $\begin{array}{l}\text { vyslovovat } \\
\text { ( } \text { prononcer }_{\text {imperf.) }}\end{array}$ & $\begin{array}{l}\text { [...] jsou plnoprávnými občany tohoto státu a mají právo vyslovovat své } \\
\text { požadavky [a svůj názor.] }\end{array}$ \\
\hline $\begin{array}{l}\text { vznášet (faire } \\
\text { envoler }\end{array}$ & $\begin{array}{l}\text { Osvětlovač nebo kameraman vznáší požadavek, aby lampa svítila více do } \\
\text { jednoho místa. }\end{array}$ \\
\hline $\begin{array}{l}\text { vznést (faire } \\
\text { envoler } \\
\text { perf. }\end{array}$ & $\begin{array}{l}\text { Ruský parlament vznesl požadavek na jednostranné zrušení sankcí proti Srbsku } \\
{[\ldots] \text {. }}\end{array}$ \\
\hline
\end{tabular}

Afin d'expliciter mieux la synonymie des différents supports, voici le dernier exemple du tableau précédent sous la forme d'un «puzzle ».

Tableau 6. - Synonymie de différents verbes support

\begin{tabular}{|c|c|c|c|}
\hline No & Vsup. & Npréd. & Compléments \\
\hline $\begin{array}{l}\text { Le parlement } \\
\text { russe }\end{array}$ & a formulé & la revendication & $\begin{array}{l}\text { d'annuler unilatéralement } \\
\text { les sanctions contre la } \\
\text { Serbie. }\end{array}$ \\
\hline \multirow{9}{*}{$\begin{array}{l}\text { Ruský } \\
\text { parlament }\end{array}$} & vznesl & \multirow[t]{7}{*}{ požadavek } & \multirow{9}{*}{$\begin{array}{l}\text { na jednostranné zrušení } \\
\text { sankcí proti Srbsku [...]. }\end{array}$} \\
\hline & dal & & \\
\hline & formuloval & & \\
\hline & předložil & & \\
\hline & přednesl & & \\
\hline & vyslovil & & \\
\hline & vznesl & & \\
\hline & přišel & \multirow[t]{2}{*}{ s požadavkem } & \\
\hline & vyrukoval & & \\
\hline
\end{tabular}

Les deux supports restants, mít (avoir) et klást (poser), réunis dans le tableau 7, ont un champ d'action plus large, en ce qu'ils peuvent remplacer n'importe quel support imperfectif du tableau 5, mais l'inverse n'est pas vrai. 
Tableau 7.

\begin{tabular}{|l|l|}
\hline Vsup. & Exemple \\
\hline mít (avoir) & $\begin{array}{l}\text { Přínosné rovněž je, že linka NOVA nemá vyšší požadavky na kvalitu skloviny } \\
\text { jako ostatní automatické linky na kalíškovinu. }\end{array}$ \\
\hline klást (poser) & Nežádáme nemožné - neklademe žádné nesplnitelné požadavky . \\
\hline
\end{tabular}

Au premier abord, la différence consiste dans le fait que mít (avoir) et klást (poser) admettent également un sujet non-humain, tandis que les supports du tableau 5 nécessitent un sujet humain. Cette propriété est couramment codée de manière binaire dans le Lexique-Grammaire, même si dans certains cas, une analyse plus fine serait nécessaire; par exemple le nom prédicatif rozhodnutí (décision) demande en tchèque deux supports différents, suivant que le sujet est une personne physique (21) ou une institution (22).

(21) Jana udělala dioležité rozhodnutí.

Jana "a fait» une décision importante.

$$
\begin{array}{ll}
\text { Komise } & \text { prijala důležité rozhodnutí. } \\
\text { La commission } & \text { a pris } » \\
\text { une décision importante. }
\end{array}
$$

Mais il nous semble pertinent de nous poser la question de savoir pourquoi les verbes du tableau 5 nécessitent un sujet humain. En effet, accompagné d'un argument-sujet «humain», le prédicat požadavek (exigence) implique nécessairement un prédicat de communication, car exiger / revendiquer quelque chose veut nécessairement dire exprimer une exigence / revendication. Ainsi, la liste des actualisateurs comporte quelques verbes de communication comme vyslovit (prononcer), formulovat (formuler), přednést (réciter), etc. D'après les exemples du corpus nous pouvons voir que dans ces verbes de communication, la fonction support et la fonction prédicative peut être plus ou moins importante, selon le contexte. Voici deux cas de figure pour le verbe přednést / přednášet

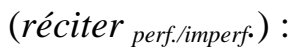

(23) Před pár týdny Státní ústav pro kontrolu léčiv přednesl svůj finální požadavek: ke konečnému schválení léku dodat studii o jeho trvanlivosti [...]. [...] l'Institut national du contrôle des médicaments a récité son exigence finale $[. .$.$] .$

(24) Živě si predstavil, jak Bob vytáčí číslo a bez slůvka vysvětlení přednáší svi̊j požadavek.

[...] Bob compose le numéro et, sans la moindre explication, récite son exigence $[\ldots]$.

La fonction support prévaut nettement dans l'exemple (23), où nous pouvons dire avec certitude que l'exigence finale de l'Institut revêtait certainement une 
forme écrite et ne pouvait pas être récitée. Par contre, dans l'exemple (24), la charge prédicative du verbe réciter est beaucoup plus importante. Dans ce contexte, le verbe réciter ne peut pas être remplacé par un verbe qui ne soit pas un verbe de communication, ce qui était possible dans la phrase (23). Ce qui est relativement gênant pour la méthode du Lexique-Grammaire, c'est que le niveau de désémantisation du verbe support dans ces cas ne semble pas dépendre d'un paramètre formel concret, ce qui rend le codage binaire difficile.

Un autre type de clivage dans le rapport entre la fonction support et la fonction prédicative peut être observé auprès des verbes qui admettent la violation de la coréférence des sujets. Cette fois-ci, la différence formelle est très nette : dès que la coréférence est violée, le verbe perd complètement la fonction support et devient pleinement prédicatif. Confrontons à ce titre l'exemple (23) où le verbe est support, avec l'exemple (25) où il est prédicatif :

(25) Starosta přednesl požadavek TS o zvýšení cen za prodej hrobového místa.

Le maire a récité l'exigence du Service d'assistance technique concernant l'augmentation des prix des emplacements au cimetière.

Dans ce cas, c'est le Service d'assistance technique qui exige, tandis que le maire ne fait que reproduire cette exigence.

Un niveau de désémantisation variable peut également être repéré dans d'autres cas qui ne concernent pas les verbes de communication. Dans les exemples du tableau 8, nous pouvons avoir une interprétation «support» ou une interprétation «prédicative » du verbe correspondant, selon le contexte de la phrase :

Tableau 8. - Désémantisation contextuelle

\begin{tabular}{|l|l|l|}
\hline V + Npréd. & Interprétation « support » & Interprétation « prédicat » \\
\hline vést jednání & être en négociation & diriger une négociation \\
\hline účastnit se jednání & être en négociation & participer à une négociation \\
\hline uplatňovat opatření & prendre des mesures & appliquer des mesures \\
\hline nabídnout opatření & prendre des mesures & proposer des mesures (à q) \\
\hline hledat námitky & avoir des objections & chercher des objections \\
\hline
\end{tabular}

Tous ces exemples laissent entendre que la désémantisation du verbe support ne doit pas nécessairement être un paramètre binaire. Un verbe support peut rester le foyer d'une prédication en fonction de certains paramètres formels, mais aussi en fonction du contexte ou - comme nous l'avons vu pour les verbes de communication - en fonction de l'accent que le locuteur met sur le sens.

Dans son article sur la fonction sémantique des verbes supports, Maurice GROSS (1998:40) aborde cette problématique par un exemple avec le verbe de communication signer :

(26) Luc a passé un contrat avec Léa.

(27) Luc a signé un contrat avec Léa. 
Le procédé est analogue, même si le verbe signer n'est pas effaçable au même titre que les verbes de communication du tableau 5, en ce qu'il serait difficile d'imaginer l'usage de l'expression signer un accord, si l'accord en question n'était pas écrit. Cependant, il s'agit là d'un autre exemple typique de la désémantisation partielle : celle qui concerne les «noms de résultat », pour lesquels nous pouvons admettre une interprétation résultative et processuelle à la fois (cf. ALONSORAMOS M., 1998: 33-34). De même, l'expression signer un contrat a un sens concret (déposer sa signature sur un papier appelé "contrat ») et un sens abstrait (conclure un accord). Le verbe signer exerce la fonction prédicative dans le premier cas et la fonction support pour le second, l'un étant indissociable de l'autre.

\section{Conclusion}

Une application systématique du test de l'effacement sur le matériel tiré du Corpus national tchèque SYN 2000 nous amène à repenser quelques aspects du modèle théorique du prédicat nominal à verbe support proposé par le LexiqueGrammaire. Les données montrent qu'une répartition binaire entre les verbes «supports» et les verbes «prédicatifs» (ou «distributionnels », dans la terminologie de Maurice Gross) est parfois trop réductrice, car le verbe appelé «support» peut remplir deux fonctions, à savoir la fonction de «support » et la fonction «prédicative » en même temps, dans une proportion variable. D'autre part, un prédicat nominal peut être actualisé par plusieurs verbes assumant la fonction du support. Ce type d'asymétrie est décrit dans l'article sur le Dualisme asymétrique du signe linguistique de Sergej KARCEVSKIJ (1964), où il est souligné qu'une forme linguistique tend à assumer plusieurs fonctions, et une fonction tend à être assumée par plusieurs formes.

En conformité avec les conclusions de F. DANEŠ (1966), il est opportun de représenter ce clivage entre la fonction (support) et la forme (verbale) correspondante en termes de relations centro-périphériques, comme il est schématisé dans le tableau suivant.

\begin{tabular}{|c|l|l|}
\hline Forme concernée & $\begin{array}{c}\text { Niveau } \\
\text { d'analyse }\end{array}$ & \multicolumn{1}{|c|}{ Fonction support plus centrale, lorsqu'il y a : } \\
\hline $\begin{array}{c}\text { (1) Verbe - pris } \\
\text { hors contexte }\end{array}$ & système & - collocabilité avec un nombre important de noms prédicatifs \\
\cline { 2 - 3 } & discours & $\begin{array}{l}\text { - rapport élevé entre le nombre d'emplois support vs. nombre } \\
\text { d'emplois non-support }\end{array}$ \\
\hline $\begin{array}{c}\text { (2) Verbe - en } \\
\text { liaison avec un } \\
\mathrm{N}_{\text {préd. }} \text { donné }\end{array}$ & système & $\begin{array}{l}\text { - neutralité stylistique du V dans la construction } \\
\text { - exclusivité de la fonction support }\end{array}$ \\
\cline { 2 - 3 } & discours & $\begin{array}{l}\text { - rapport élevé entre le nombre d'emplois support vs. emplois } \\
\text { non support (si la fonction support n'est pas exclusive) } \\
\text { - fréquence relative des différents supports possibles }\end{array}$ \\
\hline
\end{tabular}

Nous constatons que les relations centro-périphériques se manifestent à différents niveaux. (1) Pour un verbe conçu indépendamment du contexte («verbe du dictionnaire »), la fonction support sera plus centrale si le nombre des noms prédicatifs qu'il est susceptible d'actualiser est plus élevé. D'autre part, il faut prendre en compte qu'un verbe du dictionnaire peut revêtir des fonctions support et non-support (fonction prédicative, auxiliaire, etc.) dans une proportion variable ; 
toutefois, cette propriété - systémique en soi et repérable dans le dictionnaire prend de l'importance notamment si elle est observée en termes de fréquence d'usage dans le discours. Plus le nombre d'emplois en fonction support prévaut sur celui des emplois non-support, plus la fonction support est centrale pour ce verbe.

Comme un nom prédicatif est susceptible d'être actualisé par plusieurs verbes supports, il est intéressant d'appliquer la relation centro-périphérique également dans une autre perspective (2) ; ainsi, il faut considérer la centralité de la fonction support des différents verbes en relation avec un nom prédicatif donné. Ici, quatre facteurs sont à prendre en considération, dont seulement celui de la neutralité stylistique du verbe a été étudiée par le Lexique-Grammaire. Par contre, nos données montrent non seulement l'utilité du recours aux corpus de langue en vue $\mathrm{du}$ calcul relatif des fréquences, mais également une propriété systémique inattendue des constructions à support: la fonction support et la fonction prédicative ne s'excluent pas automatiquement.

\section{BIBLIOGRAPHIE}

Alonso-Ramos Margarita (1998), Étude sémantico-syntaxique des constructions à verbe-support. Thèse de Doctorat sous la direction d'Igor Mel'čuk, Université de Montréal.

CATTEL Ray (1984), Composite predicates in English. Syntax and Semantics, 17, Sydney, Academic Press Australia.

DANEŠ F., HlavSA Z. a kol. (1981), Větné vzorce v Češtině. Praha, Academia, Studie a práce lingvistické 23.

DANEŠ F. et al. (1966) Travaux linguistiques de Prague 2, Les problèmes du centre et de la périphérie du système de la langue. Prague, Academia Editions de l'Académie Tchécoslovaque des Sciences.

EFES (2004), Frekvenční slovník češtiny. [CD-ROM]. Praha, Ústav Českého národního korpusu FF UK.

GROSS Gaston (1989), Les constructions converses du français. Genève, Droz.

Gross Maurice (1975), Méthodes en syntaxe. Paris, Hermann.

Gross Maurice (1981), Les bases empiriques de la notion de prédicat sémantique. Langages 63, Paris, Larousse, p. 7-53.

HARRIS Zellig Sabbettai (1970), Elementary transformations. In: Harris Z.S., Papers in Structural and Transformational Linguistics, Dordrecht: Riedel, p. $482-532$.

IBRAHIM Amr H. (2000), Une classification des verbes en six classes asymétriques hierarchisées. In: Cordier F. et al. (ed.), Syntaxe et sémantique 2, Sémantique du lexique verbal. Caen, Presses universitaires de Caen, p. 81-98.

Karcevskij S. (1964), Du Dualisme Asymétrique du Signe Linguistique. In: Josef Vachek (compiled by), A Prague School Reader in Linguistics, Bloomington, Indiana university press, p. 81-87.

MACHÁČKOVÁ Eva (1979), Analytická spojení typu sloveso + abstraktní substantivum (analytické vyjadřování predikátů), Praha, Ústav pro jazyk český ČSAV, Kandidátská práce [thèse de $3^{\mathrm{e}}$ cycle]. 
RADIMSKÝ Jan (2005), Des méthodes de vérification en linguistique. In: Čermák Petr, Tláskal Jaromír (editores): Las lenguas románicas: su unidad y diversidad, Praha, Univerzita Karlova v Praze, Filozofická fakulta, 2005, p. 178-184.

RADIMSKÝ Jan (2009a), A propos de la voix dans la prédication nominale en italien. In.: Ibrahim A. H. (éd.), Prédicats, prédication et structures prédicatives, Paris, Cellule de recherche en linguistique, p. 162-169.

RADIMSKÝ Jan (2009b), A propos des paramètres de la prédication nominale à support en tchèque. Linguisticae investigationes, 33:2, Amsterdam, John Benjamins.

RADIMSKÝ Jan (2010), Verbo-nominální predikát s kategoriálním slovesem. České Budějovice, Jihočeská univerzita.

SYN (2000), Corpus national tchèque, base Syn 2000, [online] $<$ http://ucnk.ff.cuni.cz/>

VetUlAni Grażyna (2000), Rzeczovniki predykatywne jezyka polskiego. Poznań, Wydawnictvo naukove UAM.

\section{RIASSUNTO}

Il presente articolo fa parte di una ricerca più ampia, il cui scopo è di confrontare il funzionamento dei predicati verbonominali con verbo supporto in ceco, in italiano e in francese, sullo sfondo metodologico del lessico-grammatica di Maurice Gross. Rispetto alle pratiche di molti altri studiosi nell'ambito lessicogrammaticale, due parametri tecnici della ricerca sono stati modificati, pur rispettando i principi metodologici di origine: la ricerca è basata su grandi corpora elettronici e prende le mosse in maniera sistematica dal nome predicativo, invece di partire dal verbo supporto. Si è scoperto che la maggior parte dei nomi predicativi cechi più frequenti può essere attualizzata da più verbi supporto, tra $\mathrm{i}$ quali alcuni sono pienamente sostituibili fra di loro in certe condizioni. Confrontando sempre le possibilità teoriche del «sistema» con le realizzazioni concrete nella «parole», il presente contributo mette in dubbio la distinzione binaria tra la funzione di «supporto»e la funzione predicativa di un verbo proposta nell'ambito del lessico-grammatica. Si cerca di descrivere questo rapporto tra la forma (verbo finito) e la funzione (predicato, supporto) in termini più elastici di centro-periferia, formulati nell'ambito della Scuola di Praga. 